\title{
Corela
}

Cognition, représentation, langage

14-1 | 2016

Vol. $14, n^{\circ} 1$

\section{Le dioula véhiculaire : Situation sociolinguistique en Côte d'Ivoire}

Konate Yaya

\section{OpenEdition}

\section{Journals}

Édition électronique

URL : http://journals.openedition.org/corela/4586

DOI : $10.4000 /$ corela.4586

ISSN : $1638-573 \mathrm{X}$

\section{Éditeur}

Cercle linguistique du Centre et de l'Ouest - CerLICO

Référence électronique

Konate Yaya, «Le dioula véhiculaire : Situation sociolinguistique en Côte d'Ivoire », Corela [En ligne], 14-1 | 2016, mis en ligne le 16 juin 2016, consulté le 19 avril 2019. URL : http:// journals.openedition.org/corela/4586; DOI : 10.4000/corela.4586

Ce document a été généré automatiquement le 19 avril 2019

\section{(c) (i) (2)(2)}

Corela - cognition, représentation, langage est mis à disposition selon les termes de la licence Creative Commons Attribution - Pas d'Utilisation Commerciale - Partage dans les Mêmes Conditions 4.0 International. 


\title{
Le dioula véhiculaire: Situation sociolinguistique en Côte d'Ivoire
}

\author{
Konate Yaya
}

\section{Introduction}

1 Le Dioula véhiculaire de Côte d'Ivoire occupe en Côte d'Ivoire une place tout à fait particulière. En effet, ce parler ne peut être situé géographiquement sur une carte (Dumestre : 1971) car il est parlé sur toute l'étendue du territoire ivoirien.

2 Ce parler est différent du dioula de Bondoukou et de celui de Kong mais il est parlé et compris par tous les Mandings. C'est, en un mot, la koïnè des parlers mandings (Tera Kalilou : 1986).

3 Une enquête faite en 1968 au Lycée Classique d'Abidjan (Dumestre : 1971) a montré que plus de la moitié des élèves de ce lycée parlaient ou avaient des parents qui parlaient dioula.

4 L'implantation de cette langue va de plus en plus grandissante, puisque c'est, avec le français, la seule langue nationale véhiculaire sur l'ensemble du territoire ivoirien (Dumestre 1971).

5 Vu l'importance de cette langue en Côte d'Ivoire, nous avons décidé de faire une étude sur son rôle sociolinguistique dans ce pays. A travers cette étude, nous répondrons à ces différentes questions :

6 -quel est le rôle que joue le dioula en Côte d'Ivoire?

7 -quelle est l'attitude des différents peuples face à cette langue?

8 Tout d'abord, nous présenterons la langue dioula. Ensuite, nous présenterons le rôle sociolinguistique qu'elle joue en Côte d'Ivoire tout en exposant les attitudes des différents groupes sociaux et ethniques envers celle-ci. En outre, nous parlerons des différentes appellations que la langue peut avoir. Enfin, nous exposerons les rapports qu'entretient cette langue avec les autres. 


\section{Présentation de la langue dioula}

Avant d'aborder notre étude sociolinguistique du dioula, nous présentons tout d'abord la langue. Mais nous ne saurons parler du dioula véhiculaire de Côte d'Ivoire sans mentionner la langue manding dont elle fait partie.

En effet, la langue manding (ou mandingue, selon certaines écritures) est considérée comme la "lingua franca" (Tera Kalilou : 1983) de l'Afrique de l'ouest en raison du nombre de ses locuteurs et de sa présence dans environ douze pays de cette partie de l'Afrique à savoir : Le Burkina Faso, la Cote d'Ivoire, la Gambie, le Ghana, la Guinée, la Guinée Bissau, le Liberia, le Mali, la Mauritanie, le Niger, le Sénégal et la Sierra Leone.

Langue dominante sur le plan numérique ${ }^{1}$, statutaire et fonctionnel (elle est utilisée comme langue véhiculaire dans plusieurs pays) dans bon nombre de pays, elle occupe une place de choix dans les différentes transactions, ce qui fait d'elle une langue très importante dans la sous-région.

En Côte d'Ivoire, elle est considérée selon Dumestre : 1971 comme « la première langue véhiculaire ».

Cela s'explique d'une part, par le rôle économique qu'elle joue dans ce pays (les mandings sont commerçants et transporteurs) et d'autre part, par l'immigration massive des populations de langues mandingues des pays voisins comme le Mali, la Guinée et le Burkina Faso.

4 En effet, les commerçants et transporteurs mandings fréquentent les marchés et les routes, répandant leur langue sur toute la surface du territoire, dans les villes comme dans les banlieues (Tera Kalilou : 1986), ce qui fait qu'elle est la langue la plus répandue sur le territoire avec une expansion continue. Néanmoins, il faut souligner que les populations manding de Côte d'Ivoire viennent toutes du Mandén, haute vallée du fleuve Niger au Mali entre le XVIe et le XVIIe siècle, ce qui explique les ressemblances et les points communs à ces différents parlers. En outre, ces Mandings en arrivant en Côte d'Ivoire ont rencontré des populations déjà installées (les Senoufos au nord-ouest et à l'est, les Dans à l'ouest, les Gouros au sud et les Baoulés au sud-est).

15 La forte dialectisation de cette langue s'explique par le fait qu'elle a été en contact avec ces différents peuples parlant d'autres langues (Tera Kalilou: 1986). Différents études antérieures ont déjà permis de diviser le manding en fractions. On peut citer entre autres, les travaux de Maurice Delafosse, de Denis Creissel sans oublier Marie J. Derive. Grace aux travaux de ces chercheurs, on peut retenir que le manding peut être divisé en trois grandes fractions à savoir :

\section{-Le Malinké}

-Le Bambara

-Le dioula.

Le dioula véhiculaire de Côte d'Ivoire fait partie ainsi de la fraction dioula.

Selon Tera: 1986, le dioula véhiculaire serait né de la rencontre de différents parlers mandings du nord de la Côte d'Ivoire et aussi des pays voisins. Ces parlers mandings ivoiriens sont le wojenekakan (parler d'Odienné), le maukakan (parlé autour de Touba), le worodugukakan (parler de Seguela), le kpongakan (dioula de Kong), du jurabakan (dioula de la région de Boundiali)... Quant aux parlers mandings des pays voisins, il s'agit de ceux 
du Mali, buguninkakan de Bougouni, dioula de Sikasso et des parlers bambara de Bamako et de Ségou. Il s'agit en outre des parlers maninka de Guinée, du dioula de Bobo-Dioulasso, sans oublier le dafing burkinabé. Ces différents peuples ont été des pions essentiels au début du développement de l'économie coloniale, qui avait besoin d'une main d'œuvre abondante. Ceci a favorisé la naissance de villes peuplées de marchands qui font ensemble leurs transactions et qui attirent un bon nombre d'immigrants. C'est leur contact qui a donné naissance à ce manding commun, non seulement disponible entre eux, mais aussi entre eux et les autres groupes ethniques de la nouvelle société urbaine qui les a adoptés. Le dioula est un parler pratiqué, non seulement par des locuteurs natifs du manding, mais aussi par un grand nombre de locuteurs d'autres origines ethniques (Tera op.cit).

\section{Le sens du terme dioula}

21 Avant d'aborder notre étude sur le rôle sociolinguistique du dioula en Côte d'Ivoire, nous allons définir le sens du terme dioula qui fait aujourd'hui beaucoup de polémique.

Pour la plupart des linguistes tels que Sangaré et Tera: 2008, d'une part «Le nom dioula provient du mot manding jùla qui veut dire "marchand".

Par contre, d'autres mandingophones dont plusieurs arabisants, soutiennent que le dioula provient du terme arabe "جول " جal jûl] qui est un dérivé du terme "جولة " [jawla] de l'arabe classique qui signifie "tournée".

Cependant, tout au long de nos recherches, tous nos enquêtés traduisaient le terme « jula » par " commerçant », qui est en l'occurrence l'activité principale et majeure de ce peuple. De plus, en arabe, le mot "commerçant" est désigné par le terme " التاجر " [atâfir] qui a pour pluriel " التجار " [atifâr]. Le commerce est quant à lui désigné par 《 التجارة " [atifâra].

Pour ces arabisants, le commerçant est celui qui fait beaucoup de "tournées", d'où l'origine du terme. Toujours selon eux, dans l'histoire de la pénétration de l'islam en pays manding, pénétration qui s'est faite par le biais du commerce, les marchands mandings ont fait des échanges commerciaux avec les peuples arabes qui avaient pour intention majeure l'islamisation de cette partie de l'Afrique. C'est à la suite de ces rapports commerciaux que ces marchands mandings furent appelés "dioula", décrivant l'activité de ces marchands ambulants mandings.

D'autre part, Maurice Delafosse: 1929, avait montré que le terme dioula provenait de "jula» et signifiait "de la source, de la base" en dioula. Ainsi, (toujours selon lui), morphologiquement, nous avons : ju (la base) et la (la provenance).

Il ajouta par la suite que les "julas» expliquent leur langue comme provenant de la source ou de la base. Cette thèse nous a laissé dubitatif et nous avons décidé d'entreprendre des recherches sur la question. Après enquête, il ressort qu'un seul parmi les questionnés, d'origine de Bondoukou, ville situé à l'est de la Côte d'Ivoire nous avait répondu que le terme 'jula' signifiait "de l'origine", donc les "julas" sont les « originaires ». Dans sa réponse, il rejoint Maurice Delafosse.

Alors nous avons décidé de reprendre nos enquêtes: en effet, nos enquêtes étaient beaucoup plus orientées sur les peuples mandings du nord de la Côte d'Ivoire à savoir les wojenekas, les koyakas (parlers mandings de la fraction malinké), les julabas de Boundiali (parler manding de la fraction dioula) et même les peuples mandings des pays limitrophes 
de la Côte d'Ivoire tels que les dafings, les bougounikas et surtout les bambaras. Tous ces peuples nous disaient que le jula signifiait "commerçant" alors qu'un seul locuteur dioula de Bondoukou (ville située à l'est de la Côte d'Ivoire) nous donnait une signification semblable à celle donnée par Maurice Delafosse. Alors, nous nous sommes orientés vers les peuples mandings de cette localité (Bondoukou). Nous avons alors questionné deux personnes (des sexagénaires) qui sont des locuteurs dioulas de Bondoukou et ces derniers nous ont répondu que le terme "Jula" signifiait « commerçant ".

Ainsi donc, nous avons pu conclure que le terme "jula", emprunt de l'arabe ou pas signifie "commerçant".

\section{Rôle sociolinguistique du dioula en Côte d'Ivoire} est utilisé dans certains secteurs d'activités clés du pays (principalement le domaine du commerce et du transport). Le dioula sert aussi de langue d'échange (medium) entre les populations d'origine mandingues et aussi les populations non mandingues. (58\%). Cette hausse a été confirmée par G. Dumestre $(1971: 661)$ en ces termes : « 
L'implantation du dioula va sans doute grandissante, puisque c'est, avec le français, la seule langue véhiculaire sur l'ensemble du territoire » et S. Lafage et M. J. Derive : 1978 :

Le dioula, bien qu'appartenant à une famille linguistique parlée par seulement $14 \%$ de la population ivoirienne, a donc su conquérir, sous une forme dialectale, légèrement différente certes de sa forme originale, un rôle important comme

langue véhiculaire, celle par excellence des échanges commerciaux.

G. Dumestre (1971: 661) a insisté sur l'implantation grandissante de cette langue depuis 1971 et justifie cela par le fait que cette langue bien que nationale joue le rôle de langue véhiculaire à côté du français, langue officielle du pays.

S. Lafage et M.J. Derive (1978) ont cependant réparti les rôles véhiculaires de ces deux langues: si le français est parlé dans l'administration, l'éducation et l'enseignement supérieur, le dioula, quant à lui, est parlé dans les échanges commerciaux ainsi que dans les grandes métropoles du pays sans oublier le milieu du transport.

41 A partir de notre enquête, nous pouvons conclure que le dioula est parlé par plus de la moitié des Ivoiriens et qu'il est en expansion continue, comparé aux premières enquêtes qui ont été effectuées sur la langue.

\section{Attitudes et comportements envers la langue}

\subsection{Les mandings d'origine}

La plupart des locuteurs d'origine mandingue, qu'ils soient des immigrés maliens, burkinabés et guinéens, ou encore les peuples manding des différentes régions de la Côte d'Ivoire (les wojenekas, les koyakas, les kpongakas, ...) considèrent le dioula comme une langue dégénérée. Pour ces différents groupes manding, le dioula est un parler dégénéré issu de leur langue qu'ils considèrent comme "langue pure". Ils considèrent par ailleurs les locuteurs dioulas comme des "Tagboussi », c'est-à-dire des dégénérés (Tera: 1986). Mais cela ne s'arrête pas seulement à une considération car la langue et les locuteurs sont appelés "Tagboussi”.

Pour eux, le dioula est "forobakan", c'est-à-dire une «langue publique, commune », et lexicalement pauvre contrairement aux autres langues manding qui sont considérées comme riches lexicalement et pures.

Cependant, ils sont unanimes sur une chose: le dioula est le meilleur outil de communication entre eux et les autres groupes mandings. Mais lorsqu'ils se retrouvent entre eux, c'est-à-dire, entre ressortissants du même terroir, c'est la langue d'origine qui est utilisé, faisant alors place à une situation de diglossie.

Le dioula devient alors la langue qu'il faut utiliser entre les locuteurs de langue manding différentes et lorsqu'ils se retrouvent entre eux, c'est la langue "pure" qui est utilisée dans les conversations (familiales).

Ainsi, tous ces peuples mandings se reconnaissent à travers le dioula qu'ils considèrent comme une langue d'unité à travers laquelle tous se comprennent. 


\subsection{Les non mandings}

47 Par «non manding ", nous faisons référence aux peuples non mandings du nord de la Côte d'Ivoire, des peuples immigrés des pays limitrophes du pays ou de la zone CEDEAO (Nigeria, Niger, Togo).

La plupart de ces peuples sont des commerçants ou des manœuvres travaillant pour leur propre compte ou pour des entreprises et usines de la place. Ils ont vécu et côtoyé les "petits dioulas ${ }^{4}$ ", ce qui leur a permis de maitriser leur langue. Habitant les mêmes campements et les mêmes quartiers populaires que ces dioula, ils prient souvent ensemble dans la mosquée (la plupart d'entre eux sont des musulmans), organisent ensemble leurs cérémonies à caractère religieux et communautaires.

Pour ceux-là, la première langue d'intégration dans la société ivoirienne qui s'offre à eux est le dioula.

50 Souvent de langue maternelle du groupe gur (Senoufo, Moore, Koulango, Lobiri, Yoruba... ) et autres, ces non-mandings ont une attitude très positive envers le dioula car cette langue constitue pour eux un moyen précieux d'intégration économique et sociale au tissu ivoirien.

\subsection{Les peuples du centre, du sud, de l'est et de l'ouest ivoirien}

51 Les peuples du centre, du sud et de l'est de la Côte d'Ivoire (Akan et autres Kru du sud) considèrent le dioula comme une langue d'ouverture. D'autres par contre le considèrent comme « la langue de la racaille» (Tera: 2011) des quartiers populaires des villes et des habitants des campements de culture.

La première catégorie est constituée par les ruraux analphabètes ou d'immigrés pauvres vivant dans les mêmes quartiers et les mêmes cours communes que les "dioula" et pratiquant les mêmes petits métiers (vendeurs au marché, "balanceurs" ou chargeurs de minibus de transport en commun appelés "gbaka », mécaniciens et autres petits artisans pour la plupart analphabètes).

53 Dans cette catégorie, un certain groupe, en plus d'avoir une attitude positive envers le dioula se considère eux-mêmes comme des dioulas. Certains jeunes Akan nous avaient confié qu'ils se sentaient dans la peau des "dioulas" bien qu'étant d'origine Akan. "Nous avons vécu avec les Dioulas, grandi, mangé, fait l'enfance avec eux à tel point que nous nous sentons comme des Dioulas aujourd'hui et nous en sommes ravis. "Nous confiait un jeune akan ayant fait la majeure partie de son enfance avec les Dioulas !

Quant à la deuxième catégorie, elle est justement formée de ces exploitants agricoles qui n'ont que du mépris pour leurs métayers et ouvriers agricoles. Pour ceux-là, le dioula est un outil de communication dont on ne peut se passer pour des raisons liées aux affaires. Ils sont également en situation de diglossie et utilisent un dioula minimal (Tera: 1983) quand ils savent que le français ne suffit pas à leur besoin dans différents milieux.

Certains parmi les peuples Kru de l'ouest ivoirien et quelques Kwa du sud ont quant à eux, depuis plus d'une décennie, en Côte d'Ivoire, une autre attitude envers le dioula: le mépris, animé par la situation socio-politique que le pays a traversée. Pour cette catégorie, le dioula ne devrait pas exister (pour certains d'entre eux, le dioula n'est pas 
une langue ivoirienne). Cette catégorie a une attitude négative envers le dioula qu'il considère comme une langue étrangère et conflictuelle.

\subsection{Les arabophones}

Par arabophones, nous faisons référence aux peuples d'origine arabe et qui ont cette langue comme langue maternelle. Ce sont entre autres les Mauritaniens, les Libanais, les Syriens, les Marocains...Ces peuples (Konaté Yaya :2005), venus s'installer en Côte d'Ivoire pour les affaires sont pour la plupart des commerçants ayant des (grands) magasins de marchandises ou des boutiques situés un peu partout dans les communes d'Abidjan et les villes et villages du pays. Dans leurs activités, les peuples avec qui ils font beaucoup leurs transactions sont des Dioulas qui eux-mêmes ont pour principale activité le commerce ; la conséquence de ce contact est la recherche d'une langue servant de véhicule entre les deux parties: le dioula devient alors nécessaire et demeure le principal outil de communication qui s'impose. Ces peuples immigrés se voient dans l'obligation d'utiliser le dioula qui devient alors une langue d'intégration nationale.

Ainsi, dans les boutiques gérées par ces Mauritaniens (pour la plupart), il est très courant de voir un certain "Mohammad ould" ou "Chekna" (prénoms arabes et musulmans) s'exprimer parfaitement en dioula même si dans leur parler, on note la présence de fortes interférences (surtout phoniques).

En outre, il est très courant de voir aussi un Libanais ou un Syrien ou un Marocain dans un grand magasin de la commune d'Adjamé (commune d'Abidjan ayant une forte concentration de commerçants d'origine mandingue) converser avec des acheteuses dans un dioula plus ou moins compréhensif (présence d'interférences phoniques et lexicales).

Ces différents peuples arabophones ont une attitude positive envers le dioula qu'ils considèrent comme une langue d'intégration nationale. Parler cette langue signifie pour eux qu'ils sont inscrits dans le tissu social ivoirien.

\section{5. dioula, malinké ou bambara : les différentes appellations de la langue}

60 Le dioula est tantôt appelé 'dioula', 'malinké' ou 'bambara' et cela en fonction du sentiment des locuteurs eux-mêmes pour la langue. En effet, le nom courant de la langue et la plus connue est "dioula" comme l'illustre l'exemple dans la langue elle-même : ' $n$ be julakan fo', Je parle dioula. Ce terme est accepté par presque tous les locuteurs d'origine mandingue ou même d'autres locuteurs d'origines différentes telles que les peuples du sud, du centre, de l'est et de l'ouest de la Côte d'Ivoire (Akan, Kwa ou Kru) sans oublier les peuples non manding du nord de la Côte d'Ivoire qui ont attitude positive envers la langue (Lobiri, Syennari, Koulango...). A ces peuples ivoiriens, nous pouvons ajouter les peuples immigrés tels que les Yorubas, les Mossis, les Bissas...

61 Pour tous ces peuples, le nom de la langue est le "dioula". Quant au terme Tagboussi, il est donné et utilisé par les peuples d'origine manding de la Côte d'Ivoire (les koyakas, les wojenekas...) et les peuples immigrés d'origine mandingue (Bambaras, Maninkas, Bougounikas...) pour montrer leur mépris pour un parler qui selon eux, est dégénéré et qui aurait perdu son authenticité. 


\section{le nom “malinké' à la langue. Ce terme est dérivé de 'maninkakan' et est considéré comme} une fraction de la langue mandingue selon Maurice Delafosse : 1929, Denis Creissels : 1979 et M.J Derive : 1981 au même titre que les fractions 'dioula' et 'bambara'.

Cette appellation par les intellectuels francophones ivoiriens se justifie par une certaine gêne face au terme 'dioula' qui selon eux est une langue populaire et de la racaille. Cette appellation est aujourd'hui la plus communiquée car les medias ivoiriens diffusent ce terme et on rectifie même ceux qui prononcent le terme 'dioula'. Dans les informations en langues nationales diffusées sur la Radiodiffusion Télévision Ivoirienne (RTI), c'est le terme Malinké qui s'affiche devant les écrans. Cette appellation tellement propagée dans la Côte d'Ivoire fait que si quelqu'un utilise le terme 'dioula' pour parler de la langue, il est automatiquement arrêté et renvoyé vers le terme 'malinké'.

Un jeune dioula de Bondoukou ${ }^{5}$ me racontait ceci : «je suis allé au centre de transfusion sanguine à Treichville (commune de la ville d'Abidjan) pour un don de sang, la femme qui est chargée de recenser les informations me demande mon groupe ethnique. Je lui réponds que je suis Dioula et elle dit " non, tu n'es pas dioula" mais tu es Malinké. Sa réponse ne me plut pas, alors je lui imposai le terme "dioula" en lieu et place de "malinké".

Le témoignage de ce jeune homme est une pratique courante en Côte d'Ivoire car partout dans les institutions, les secteurs d'activités, les milieux intellectuels, c'est le terme 'malinké' qui est utilisé et il est imposé dans les autres milieux pour faire disparaître le mot 'dioula' qui, selon eux n'existe pas.

Tera (1986: 6) soutient qu'il y a chez ces intellectuels un véritable déchirement entre une origine et une identité qu'ils s'entêtent à nier ou à anoblir par un terme pour eux noble, Malinké. Cependant, tout le monde n'accepte pas ce terme comme ces intellectuels voudraient que cela soit.

Quant aux professionnels de la communication manding (les griots et les imams), ils utilisent le terme "bamanankan" qui est l'équivalent du terme 'bambara'. Pour ces professionnels de la communication, qui utilisent une forme soutenue du dioula qu'est le bambara, il faut anoblir le terme en le rapprochant de la langue bambara qui est considéré comme une langue 'pure' et ayant un champ lexical riche, contrairement au dioula.

\section{6-Les rapports entre le dioula et d'autres langues}

\subsection{Les rapports entre le dioula et le français}

68 Kouadio N'guessan présentait en 2007 un article sur les rapports en le français et le dioula. En effet, il est bon de rappeler que les deux langues véhiculaires de la Côte d'Ivoire ne sont autres que le français et le dioula. L'histoire de leur implantation présente quelques similitudes. Toujours selon Kouadio : 2007, le dioula a précédé le français sur le territoire ivoirien. Après la chute de l'empire du Mali vers le 15eme siècle, les populations mandings ont commencé à essaimer dans toute l'Afrique de l'ouest. Ces populations mandings qui n'avaient d'autres activités que le commerce colportaient avec eux, en même temps que leurs marchandises, leur langue et leur religion (Tera: 1986). Même avec l'avènement de la colonisation, le dioula continuera à être influent et continuera son 
expansion. C'est à ce moment que le dioula va être en contact avec le français. En effet les négociants européens trouvent que les dioulas sont des auxiliaires précieux. En retour, les Dioula, en suivant ces derniers, voient s'ouvrir devant eux la route des zones littorales jusque-là impénétrables à cause de l'écran de la grande forêt. On voit alors des Dioula se répandre dans la forêt la plus profonde, dans les plus petits hameaux, achetant des produits et proposant leurs marchandises ; et dans le morcellement linguistique extrême de ces zones, le dioula s'impose tout de suite comme langue de communication interethnique.

Ce phénomène va s'accélérer avec le développement des villes modernes qui vont constituer pour toutes les populations ivoiriennes des zones d'attraction.

Les activités naturelles du dioula le poussent donc vers les villes.

71 Ainsi le français et le dioula se sont croisés très tôt et cette cohabitation s'est très vite soldée par une influence réciproque, matérialisée principalement au niveau lexical par des emprunts. Quelques exemples d'emprunts du dioula au français :

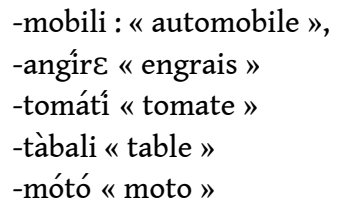

Quant aux emprunts du français au dioula, on peut citer les termes suivants qui ont, pour certains, intégré le lexique du français ivoirien depuis des lustres :

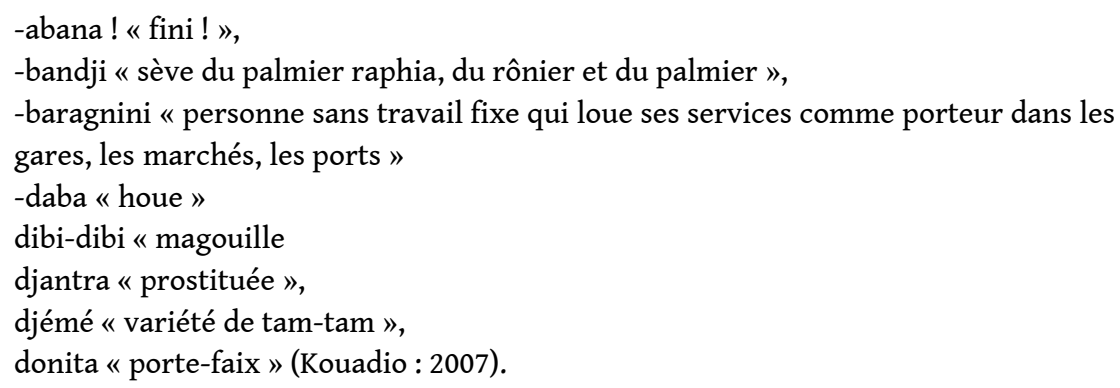

73 Cette influence est facilement compréhensible du fait que les deux langues sont liées à l'urbanisation et aux secteurs d'activités modernes. Nous pouvons sans doute affirmer que le dioula et le français apparaissent comme les deux seules langues véhiculaires de la Côte d'Ivoire.

\subsection{Les rapports entre le dioula et le nouchi}

Pour mieux parler des rapports entre le dioula et le nouchi, il faut revenir à l'étymologie du terme. En effet, selon Kouadio : 2007, l'origine du terme «nouchi » provient d'un mot composé à partir de deux mots dioula, l'un "nou » signifiant «nez " et l'autre "chi " "poil ». D'autres par contre soutiennent que l'origine du terme 'nouchi' vient de la langue susu. Cependant, s'il y a une chose à laquelle tous les sociolinguistes ivoiriens sont unanimes, c'est que les langues sources du nouchi sont ces deux parlers mandingues : le susu et le dioula. Pour certains, le nouchi serait né à Treichville ${ }^{6}$. D'autres par contre, tel que Kouadio : 2007 soutiennent que le nouchi serait né à Adjamé. En conservant cette hypothèse, on se rend compte que la commune d'Adjamé est l'une de celles où la population d'origine mandingue est supérieure à celles d'autres communautés. Le 
recensement général de la population de la commune d'Adjamé ${ }^{7}$ en mai 2014 donne les chiffres suivants :

Adjamé : $372.978^{8}$ dont $39 \%$ de Mandé.

L'on sait également que cette population a un taux d'urbanisation (ancienne) très élevé. En 1998 par exemple, le taux d'urbanisation des populations mandingues était de 61,90\%, au niveau du pays tout entier, contre 47,05 \% de Kru, 39,63 \% d'Akan (ou Kwa) et 32,27 \% de Gur. Ainsi donc, de tout temps, le concurrent direct du français a été le dioula (Kouadio : 2007), « koïnè qui permet aux mandingues d'origines diverses de communiquer entre eux et avec les non mandingues» (Tera : 1986).

C'est de cette concurrence qu'est né le nouchi (Kouadio : 2007). Cela a pour conséquence immédiate, l'infiltration des mots dioulas dans le nouchi. En effet, comme le souligne Kouadio : 2007, dans ces milieux marginaux, les jeunes dioulas sont les plus nombreux et leur langue, le dioula, s'incruste abondamment dans le nouchi. C'est ce qui explique qu'au départ, plus de la moitié du vocabulaire nouchi provenait du dioula (Kouadio : op.cit).

Aujourd'hui, le nouchi continue d'évoluer et les nouveaux locuteurs utilisent de moins en moins le dioula dans leur vocabulaire. On comprend par-là que l'usage du lexique du nouchi dépend de l'origine sociale et le niveau de scolarisation des locuteurs. Les étudiants et les élèves ont par exemple moins de mots dioulas nouveaux dans leur vocabulaire. Quoi qu'il en soit, le dioula reste encore, et de loin, la première langue (parmi les langues ivoiriennes) pourvoyeuse de mots au nouchi (Kouadio: 2007), comme l'illustrent les exemples ci-dessous :

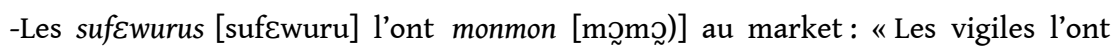
fouillés au marché »;

-Tout est nyagami [nagami] : « Tout est melangé » ;

-Il a fangan [faga] : « il a de la force »;

Il y a gbangban [gbagha] à l'université : «il a des problèmes à l'université en ce moment»,

-Il a déjà nyimi kaba : « elle a déjà fait de la prison »;

-Donnes moi gbese : « donnes moi cinq cents francs ».

Les mots suivants en italique sufEwurus, monmon, nyagami, fangan, gbangban, nyimi kaba, gbesc sont empruntés directement du dioula.

Les locuteurs du nouchi utilisent aussi les suffixes dioula -ya, -li, -ko pour créer des mots par suffixation comme dans :

janjouya : « prostitution », de janju « prostituée » + ya ;

dabali : «nourriture », de daba «manger »+li; ce terme est synonyme de badouko « nourriture », de badou « manger » + ko.

3 Une remarque importante : le nouchi emprunte les suffixes dérivatifs principalement à trois langues : deux européennes, le français (9 suffixes) et l'anglais (2 suffixes) et une seule langue ivoirienne qui n'est autre que le dioula (4 suffixes). Mais la part du vocabulaire à base de dioula baisse lorsque le nouchi est parlé par les élèves et les étudiants (Kouadio : 2007).

\section{Conclusion}

84 Notre présent article avait pour but de présenter le rôle sociolinguistique d'une langue de Côte d'Ivoire : le dioula véhiculaire. En effet, le dioula joue un très grand rôle dans le pays. 
Plusieurs études entreprises sur la langue ont montré que le dioula était parlé par plus de la moitié des ivoiriens, ce qui fait d'elle, la seule langue véhiculaire sur l'étendue du territoire ivoirien. Différentes enquêtes menées auprès des populations ont montré que ces derniers ont une attitude positive envers la langue. En effet, les mandings d'origine ont une attitude puriste envers la langue qu'ils considèrent comme une langue dégénérée, pauvre lexicalement. Cependant, ils reconnaissent que c'est le meilleur outil de communication qui s'offre à tous. Quant aux autres peuples non mandings, ils ont une attitude positive envers le dioula qu'ils considèrent comme une langue d'ouverture et d'intégration même si certains la répugnent.

En outre, nous avons présenté les différents noms de la langue. En effet, le dioula est tantôt appelé 'dioula', 'malinké' ou 'bambara' selon le sentiment des locuteurs envers la langue. Pour l'administration et les intellectuels, c'est le 'malinké'. Quant aux griots et imams mandings, c'est le nom 'bambara' qui sied le mieux. Pour les autres locuteurs, qui sont les plus nombreux, le nom de la langue est 'dioula'.

Enfin, nous avons présenté les différents rapports que le dioula entretient avec d'autres langues à savoir : le français, la langue officielle du pays et le nouchi.

En effet, les rapports entre le dioula et le français remontent à une époque éloignée et cela s'est soldé par l'emprunt réciproque des mots des deux langues

Quant aux rapports entre le nouchi et le dioula, il ressort au terme de notre étude que le nouchi s'est constitué à partir de la base du français avec des compositions des différentes langues nationales. Cependant, le dioula demeure, de loin comme de près, la première langue, parmi les langues locales, pourvoyeuse de mots au nouchi.

\section{BIBLIOGRAPHIE}

Creissels D., (1979), « Variations dialectales dans les systèmes de marques prédicatives des parlers mandings ", Publication de l'université des langues et lettres de Grenoble, Grenoble.

Delafosse M., (1929), « La langue mandingue et ses dialectes », librairie Geuthnar, Tome I, Paris.

Dérive M.,-J., (1981), « Variations dialectales des parlers mandings de Côte d'Ivoire », in Mandeukan, $\mathrm{n}^{\circ} 1$.

Derive J., et M-J., (1986), « Francophonie et pratique linguistique en Côte d'Ivoire », Politique africaine, Côte d'Ivoire.

Derive M.-J., et Lafage S, (1978), « La Côte d'Ivoire (situation sociolinguistique), Inventaire des études linguistiques sur les pays d'Afrique noire d'expression française et sur Madagascar ", CILF, Paris.

Dumestre G., (1971), « Le dioula », Actes du huitième congrès de la société linguistique de l'Afrique occidentale, volume 2, Annales de l'université d'Abidjan, série H.

Konaté Y., (2005), « Un aperçu du manding parlé par les arabisants et les arabophones de Côte d'Ivoire », Mémoire de Maitrise, ILA. 
Konaté Y., (2008), « Essai sur le verbe du dioula de Côte d'Ivoire » : Rapport de DEA, ILA.

Kouadio N'guessan J., (2006), « Le nouchi et les rapports dioula-français » dans Le français en Afrique ", revue des observatoires du français contemporain en Afrique, $n^{\circ} 21 \mathrm{CNRS}$.

Sangaré A., (1984), « Dioula de Kong (Côte d'Ivoire) : phonologie, grammaire, lexique et textes » Grenoble : université de Grenoble III, 1984, doctorat de troisième cycle : linguistique.

Sanogo Mamadou Lamine (2006) : « La recherche terminologique dans un dialecte ouvert : le cas du dioula », CNRST, Burkina Faso

Tera K., (1986), « Le Dioula Véhiculaire de Côte-d'Ivoire : Expansion et Développement », CIRL n $\circ$ 20, ILA Abidjan.

Tera K., (1983), « Tendances phonologiques et syntaxiques dans le dioula de Côte d'Ivoire », tiré de l'ouvrage Etude sur le manding de Côte d'Ivoire de Braconnier Cassian / Cassian Braconnier, John Maire, Kalilou Tera, Abidjan, ILA, AGECOOP.

Tera, K., (2011), « Attitudes envers le dioula et pratiques en Côte d'Ivoire » publié sur http // llacan.vjf.cnrs.fr/fichiers/mande2011/bublio/tera.pdf

\section{NOTES}

1. D'après les chiffres de Platiel (1978: 48), plus de quatre millions de personnes en Afrique Occidentale sont locuteurs natifs d'une langue manding. Grimes : 1996 portait le nombre à plus de huit millions.

2. La première a été menée en 1968 et avait montré que 51 \% des questions parlaient ou avaient des parents qui parlaient dioula.

3. Malheureusement, nous n'avons que les pourcentages de l'étude menée en 1968 sur le dioula.

4. Appellation de la jeunesse d'origine manding en Côte d'Ivoire

5. Ceci est un témoignage d'un jeune manding qui fait partie de l'une des motivations qui nous ont poussées à faire une étude sociolinguistique sur le dioula véhiculaire de Côte d'Ivoire.

6. Commune de la ville d'Abidjan

7. Commune de la ville d'Abidjan

8. Sources : INS, RGHP du 15 mai 2014

\section{RÉSUMÉS}

Notre présent article se propose de présenter le rôle que joue le dioula véhiculaire en Côte d'Ivoire. En effet, bon nombre d'études ont été déjà faites sur la langue en question, montrant à quel point elle est dynamique et importante tant bien économiquement que socialement. Ce présent article a pour but de montrer cette place particulière qu'a cette langue à travers différentes enquêtes menées auprès des populations ivoiriennes.

Our present article intends to present the role of vehicular dioula in Côte d'Ivoire. Indeed, many studies have already been made on the language in question, showing how the language is 
dynamic and important as well as economically and socially. The present article aims to show that special place that has this language through different surveys Ivorian people.

INDEX

Mots-clés : Dioula, sociolinguistique, rôle, langue, véhiculaire.

Keywords : sociolinguistics, language, vehicular.

\section{AUTEUR}

\section{KONATE YAYA}

Université Félix Houphouët Boigny

konatyay60@yahoo.fr 